\title{
Fluides quantiques de lumière dans les microcavités à semi-conducteurs
}

Jacqueline Bloch (jacqueline.bloch@|pn.cnrs.fr) et Alberto Amo (alberto.amo@|pn.cnrs.fr)

Laboratoire de Photonique et de Nanostructures, LPN/CNRS, Route de Nozay, 91460 Marcoussis

Les microcavités à semi-

conducteurs permettent

de confiner dans un très petit

volume à la fois les excitations

électroniques et la lumière.

L'interaction lumière-matière

s'y trouve si fortement

renforcée que les états

quantiques qui régissent

leurs propriétés physiques

sont des quasi-particules

mi-lumière mi-matière,

appelées polaritons de cavité.

Ces polaritons sont des

bosons ; ils se propagent

comme des photons, mais

interagissent fortement entre eux et avec leur environnement,

grâce à leur partie matière.

En sculptant les microcavités, nous développons

une nouvelle plateforme

pour l'étude et la manipulation des condensats bosoniques,

et pour la simulation

de différents systèmes

physiques non linéaires.
L'émission spontanée de lumière n'est pas une propriété intrinsèque de l'émetteur, mais peut être fortement modifiée lorsque l'on change son environnement électromagnétique. Lorsque l'interaction lumièrematière est suffisamment renforcée, par exemple en plaçant l'émetteur au sein d'une cavité optique, un photon émis peut être réfléchi puis réabsorbé très rapidement, avant d'avoir eu le temps de s'échapper de la cavité. Il est ensuite réémis, réabsorbé et ainsi de suite. Les états propres du système sont alors des états mixtes lumière-matière. Ce régime, dit de couplage fort lumièrematière, a été observé expérimentalement en 1992, d'une part pour des vapeurs atomiques en cavité et, d'autre part, en optique des solides pour des semi-conducteurs insérés dans une microcavité optique. Dans ce dernier cas, les états mixtes lumière-matière sont appelés "polaritons de cavité " (encadré 1).

Une avancée majeure de la physique atomique a été la démonstration, en 1995, de la condensation de Bose-Einstein d'un nuage d'atomes de rubidium, lorsque celui-ci est refroidi en dessous d'une température critique. Au sein du condensat, tous les atomes occupent le même état quantique. Le nuage atomique se comporte alors comme une onde macroscopique cohérente, illustration magnifique de la dualité onde-corpuscule. En 2006, un nouveau parallèle entre physique atomique et microcavités à semi-conducteurs est établi avec la démonstration de la condensation de Bose des polaritons de cavité. La recherche sur les condensats de polaritons, à la fois théorique et expérimentale, a connu ces dernières années un essor considérable, et des propriétés fascinantes, comme la superfluidité, la formation de vortex ou encore une cohérence spatiale macroscopique, ont été mises en évidence.

La physique des cavités à semi-conducteurs est ainsi très proche de celle des atomes froids, mais avec l'avantage crucial de faire appel à des montages expérimentaux beaucoup plus simples et facilement reconfigurables. La masse très légère des polaritons $\left(10^{-5}\right.$ fois celle d'un électron) permet d'en former des condensats à des températures huit ordres de grandeur plus élevées que leurs frères atomiques (10 à $300 \mathrm{~K}$ selon les matériaux). De plus, la dissipation, qui est une propriété spécifique des polaritons liée à la perte constante de photons à travers les miroirs, donne naissance à de nouveaux effets physiques qui sont étudiés en profondeur aujourd'hui.

Notre groupe de recherche au Laboratoire de Photonique et de Nanostructures a mis à profit les technologies de nanofabrication pour réduire la dimensionnalité des microcavités à semi-conducteurs, et les sculpter dans des géométries complexes. Dans cet article, nous proposons d'illustrer par quelques exemples la richesse de la physique que l'on peut explorer grâce à ce système photonique. Nous rappellerons tout d'abord les principales propriétés physiques des polaritons de cavité. Nous décrirons ensuite comment il est possible de générer et manipuler des flux de polaritons cohérents dans des circuits et d'observer leur superfluidité. Enfin, nous montrerons que l'on peut réaliser des potentiels complexes pour les polaritons et utiliser cette plateforme photonique comme un « émulateur quantique ». 


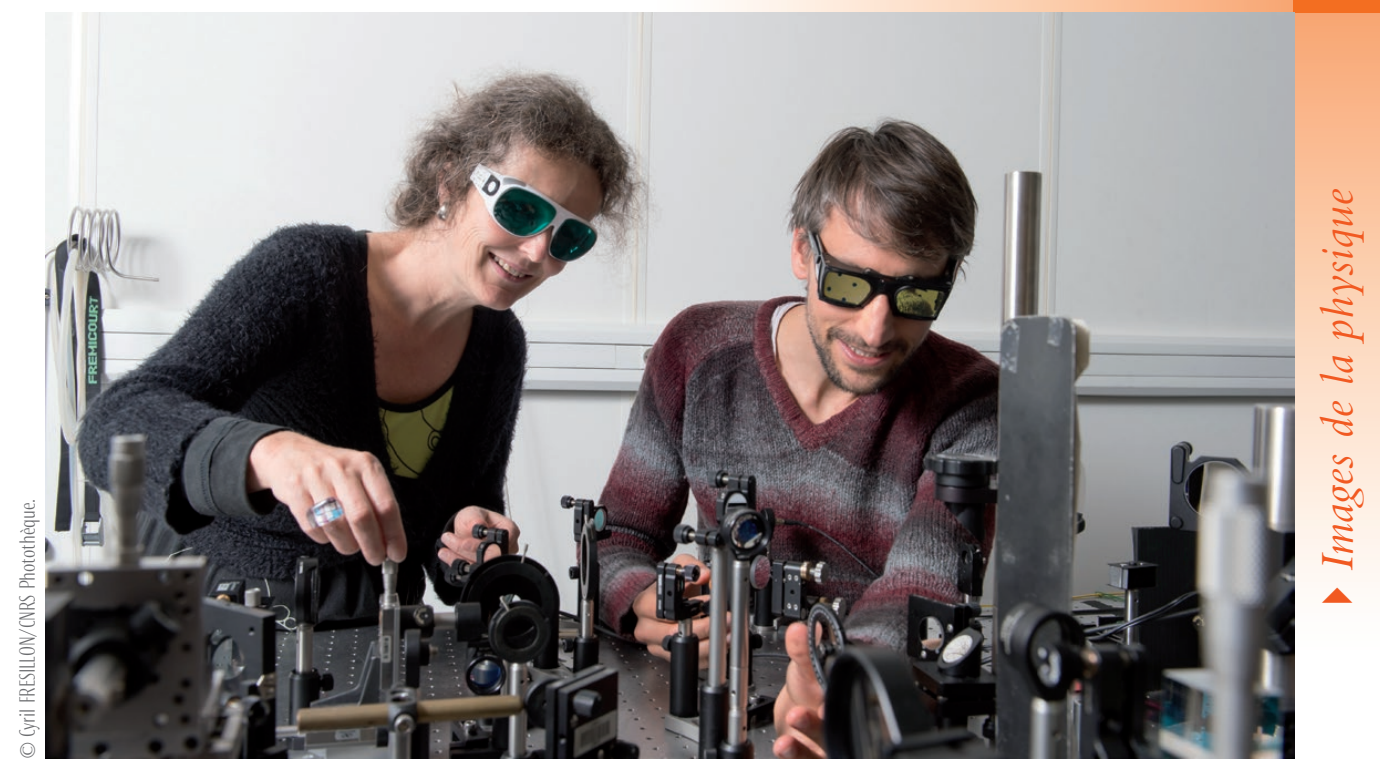

Alberto Amo (à droite) et Jacqueline Bloch mènent une expérience d’optique pour créer et manipuler un condensat de polaritons.

\section{Propriétés physiques des polaritons de cavité}

Comme décrit dans l'encadré 1, les polaritons de cavité sont les quasi-particules hybrides lumière-matière issues d'une oscillation cohérente d'un quantum d'énergie entre un exciton (une excitation électronique du semi-conducteur) et un photon. L'exciton est une paire électrontrou liée par interaction coulombienne qui peut s'annihiler au bout d'une durée caractéristique (typiquement quelques picosecondes) en émettant un photon qui, après avoir oscillé dans la cavité, peut être absorbé à nouveau et recréer un exciton et ainsi de suite. Cette oscillation cohérente est responsable de la formation de ces états propres hybrides lumière-matière.

Les propriétés physiques des polaritons de cavité sont intimement liées à leur double nature exciton-photon.

De leur partie photonique, les polaritons de cavité acquièrent des propriétés de propagation proches de celles de la lumière. La partie photonique du polariton est également responsable de dissipation dans le système, liée à l'échappement du photon hors de la cavité au travers des miroirs (un polariton est alors détruit dans le système). Ces pertes seront d'autant plus faibles que le facteur de qualité de la cavité est élevé. Nous tirons profit de ces photons qui s'échappent de la cavité pour mesurer toutes les propriétés physiques des polaritons en son sein. Ainsi, un photon émis par un polariton de vecteur d'onde $\mathrm{k}$ va sortir de l'échantillon selon un angle $\theta$ directement lié à $\mathbf{k}$. Des expériences de photoémission résolue en angle (figure E1b de l'encadré)

\section{Microcavités optiques à semi-conducteurs}

\section{et polaritons de cavité}

La structure typique d'un échantillon contenant une cavité bidimensionnelle à semiconducteurs est présentée sur la figure E1a. L'échantillon est réalisé par croissance cristalline sous ultravide (épitaxie par jets moléculaires). La structure comporte un empilement de couches de semi-conducteurs $\mathrm{Ga}_{\mathrm{x}} \mathrm{Al}_{1-\mathrm{x}} \mathrm{As}$ de différentes compositions (donc d'indices optiques différents) qui réalisent deux miroirs interférentiels entourant une cavité optique. On définit ainsi une cavité Fabry-Pérot avec des modes optiques confinés, dont l'énergie en fonction de l'angle d'émission $\theta$ varie comme indiqué sur la figure E1b (courbe Photon en tirets rouges). On voit qu'il est possible de décrire la dispersion du mode optique au voisinage de l'impulsion nulle $\left(\mathbf{k}_{/ /}=0\right)$ par une parabole. On peut donc associer aux photons confinés dans la cavité une masse effective ! Cela signifie simplement que l'énergie cinétique du photon dans le plan de la cavité est similaire à celle d'une particule massive, mais extrêmement légère $\left(10^{-5}\right.$ fois la masse de l'électron).

Aux ventres du champ électromagnétique de ce mode optique, on positionne des couches planes minces de semi-conducteurs, parallèles aux miroirs, qui réalisent des puits quantiques bidimensionnels pour les électrons (fig. E1a). Lorsque ces puits quantiques sont excités optiquement, des paires électron-trou liées par interaction coulombienne, appelées " excitons ", sont photocréées. Ces excitons sont l'excitation électronique élémentaire du système. Ils peuvent se recombiner (et donc disparaître) en émettant un photon.

Les polaritons de cavité sont les états hybrides exciton-photon issus de l'oscillation de l'énergie entre exciton et photon : un exciton disparaît pour donner naissance à un photon, qui est à son tour absorbé pour donner naissance à un exciton, etc. Les niveaux d'énergie de polaritons présentent deux branches, comme montré sur la figure E1b.
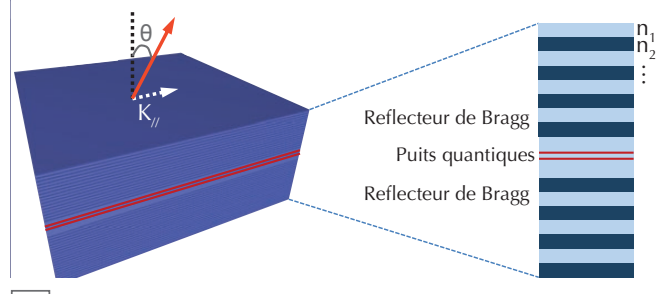

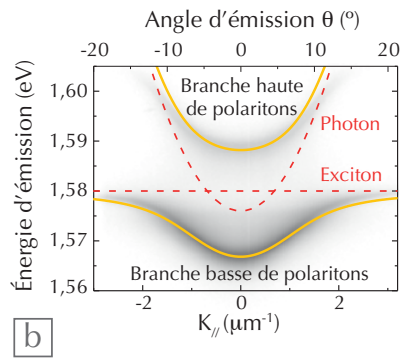

$\mathrm{b}$
E1. (a) Schéma d'une microcavité semi-conductrice bidimensionnelle contenant deux puits quantiques. $n_{1}$ et $n_{2}$ sont les indices optiques des couches de semi-conducteurs constituant les miroirs. (b) Dispersion des photons confinés dans la cavité et des excitons (courbe et droite en tirets rouges), ainsi que des polaritons (traits pleins). La dispersion polaritonique est accessible dans une expérience de photoluminescence, comme montré ici en gris (un signal plus intense correspond à une région plus foncée). L'angle d'émission $\theta$ d'un photon (flèche rouge sur le schéma a) est lié de façon univoque au vecteur d'onde $\mathbf{k}_{/ /}$des polaritons dans le plan de la cavité 


\section{\>}

permettent ainsi de mesurer directement l'énergie des polaritons en fonction de leur impulsion $\hbar \mathbf{k}$ et leur distribution dans l'espace des impulsions.

Grâce à leur composante excitonique, les polaritons de cavité interagissent avec leur environnement. En effet, il existe de fortes interactions, d'origine coulombienne, entre excitons : bien que particules " neutres ", les excitons sont formés par une paire de porteurs de charges, électron et trou. Les interactions exciton-exciton sont dominées par un terme d'échange, qui est répulsif. Ainsi, via leurs parties excitoniques, deux polaritons se repoussent. Cette interaction polariton-polariton donne lieu à des non-linéarités optiques de type Kerr géante. Un polariton peut également subir une interaction répulsive avec des excitons : ceci donne un levier pour manipuler des flux de polaritons, en injectant des excitons de façon contrôlée sur leur trajectoire.

Enfin, comme les excitons et les photons sont tous deux des bosons, les polaritons obéissent à une statistique bosonique. Il est donc possible d'accumuler un très grand nombre de ces quasi-particules dans un même état quantique et de former ainsi un fluide quantique fortement non linéaire.

\section{Gaz unidimensionnels de polaritons, de la condensation bosonique à la gravitation analogue}

Dans cette section, nous allons montrer comment on peut générer des flux de polaritons cohérents unidimensionnels, manipuler optiquement ces fluides quantiques et observer leur superfluidité.

En utilisant les techniques bien maitrisées de la nanofabrication que sont la lithographie électronique et la gravure par bombardement ionique, nous gravons les microcavités à semi-conducteurs sous la forme de rubans unidimensionnels (cavités 1D), de micropiliers ou encore de réseaux de micropiliers couplés. La distance entre les microstructures $(\sim 20 \mu \mathrm{m})$ est suffisante pour qu'elles soient totalement découplées, et nous les étudions individuellement. La figure 1a montre l'image en microscopie électronique à balayage de plusieurs cavités 1D dont la dimension transverse est de l'ordre de quelques microns. La différence d'indice optique entre l'air et le semi-conducteur induit un confinement latéral du mode optique et donc des états polaritoniques.
Formation de condensats de polaritons

Un condensat de polaritons est généré en excitant par le dessus une petite région d'une cavité 1D grâce à un faisceau laser focalisé, dont l'énergie est supérieure à celle des polaritons (on parle d'excitation non résonante). Au-dessus d'un seuil de puissance d'excitation, une émission très brillante et monochromatique apparait, qui révèle la formation d'un condensat de polaritons (fig. 1b). Les polaritons s'accumulent massivement dans l'état de plus basse énergie et nous détectons les photons qui s'en échappent. Étonnamment, l'émission très brillante s'étend très loin de chaque côté de la zone d'excitation, comme le montre la figure $1 \mathrm{~b}$. Pour comprendre cette expansion spatiale, il faut se rappeler que l'excitation optique crée localement un nuage d'excitons non condensés. L'interaction répulsive entre ces excitons et les polaritons induit localement une "colline " de potentiel pour les états de polaritons. Les polaritons générés au sommet de cette colline subissent une accélération qui les éjecte de la zone d'excitation et les conduit à se propager de part et d'autre. C'est ce qui explique l'expansion du condensat, observée sur toute la longueur de la cavité (200 $\mu \mathrm{m}$ dans cette expérience).

\section{Cohérence spontanée}

Une signature caractéristique de la condensation de Bose est l'apparition d'une cohérence spontanée. De façon remarquable dans l'expérience décrite ici, la cohérence spontanée du condensat est

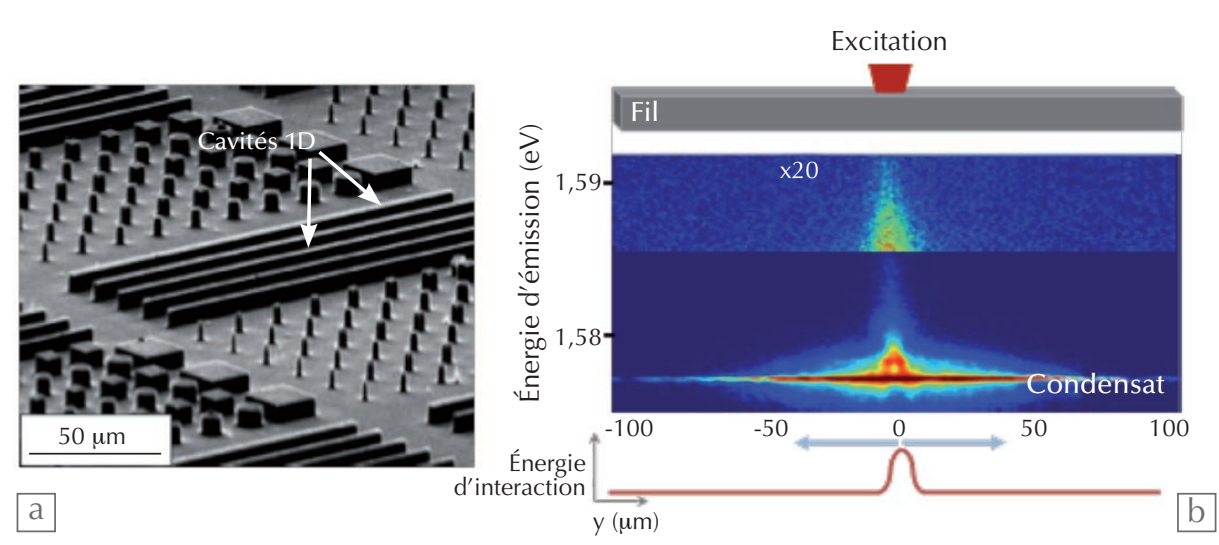

1. Condensats de polaritons dans une structure 1D.

(a) Image en microscopie électronique à balayage de plusieurs microstructures à polaritons gravées à partir d'une microcavité planaire. Deux cavités 1D sont indiquées par des flèches.

(b) Condensation des polaritons observée dans une cavité unidimensionnelle de 3 microns de largeur, excitée en son centre ; l'interaction avec le nuage d'excitons non condensés crée une « colline » de potentiel (représentée en bas, courbe rouge), qui accélère le condensat vers les deux extrémités de la cavité $1 \mathrm{D}$.
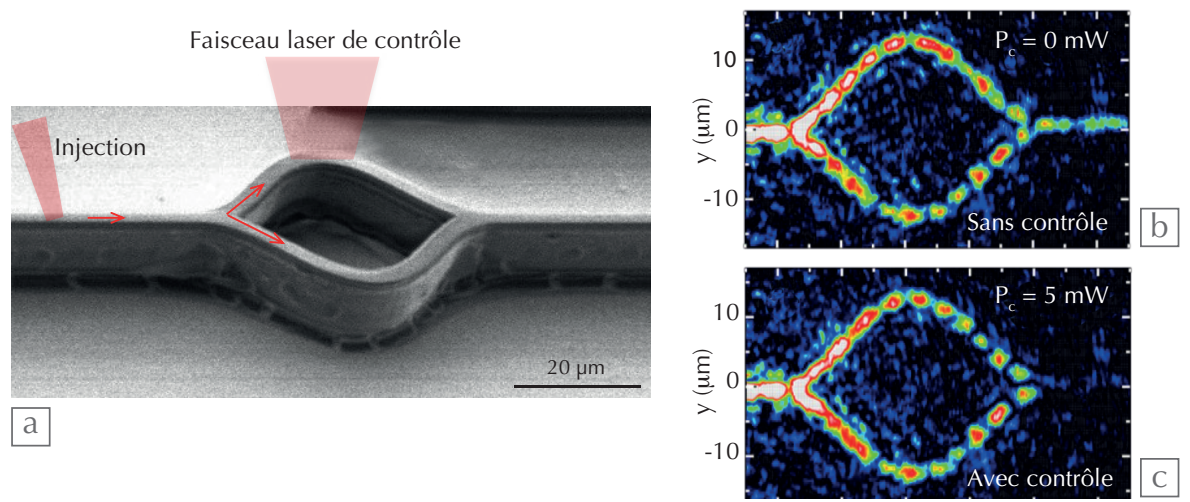

2. Un interféromètre à polaritons.

(a) Image en microscopie à balayage de l'interféromètre à polaritons.

(b-c) Images observées en collectant le signal sortant de l'interféromètre par le dessus. En (b), les polaritons interfèrent de manière constructive dans le bras de sortie ; en (c), une différence de phase de $\pi$ entre les deux bras est introduite grâce au faisceau de contrôle : une interférence destructive se produit dans le bras de sortie et annule la transmission du dispositif. 
parfaitement préservée au cours de son expansion. Ceci est démontré en réalisant une expérience de type trous d'Young, dans laquelle nous prélevons uniquement l'émission des deux extrémités de la cavité 1D et faisons interférer ces deux faisceaux lumineux. Nous observons des franges d'interférence très prononcées qui montrent la cohérence relative entre quasi-particules distantes de $200 \mu \mathrm{m}$.

Cette expérience, réalisée en 2010, a montré que l'on peut propager de manière balistique des fluides quantiques de polaritons sans affecter leur cohérence. Elle a ouvert la voie à la réalisation de circuits photoniques dans lesquels des flux cohérents de polaritons sont propagés et manipulés optiquement.

Un exemple réalisé récemment dans notre groupe est l'interféromètre de Mach Zehnder montré sur la figure $2 \mathrm{a}$. Un flux de polaritons monochromatiques est injecté dans le bras d'entrée de l'interféromètre. Ce flux se scinde en deux à l'entrée de l'anneau : les polaritons se propageant dans les deux bras de l'interféromètre se rejoignent dans le bras de sortie. Comme les deux bras ont la même longueur, ils interferent de manière constructive et la transmission $\mathrm{du}$ dispositif est maximum (fig. 2b). Il est possible de contrôler optiquement la phase des polaritons se propageant dans l'un des deux bras en créant localement dans ce bras un nuage d'excitons, grâce à un faisceau laser de contrôle de faible intensité. Ce nuage induit une barrière de potentiel qui ralentit localement le flux de polaritons, et introduit ainsi une différence de phase par rapport aux polaritons se propageant dans l'autre bras. La figure $2 \mathrm{c}$ montre le cas où le faisceau de contrôle induit une différence de phase de $\pi$ entre les deux bras : les interférences dans le bras de sortie sont alors destructives et la transmission du dispositif tend vers zéro.

La force de la non-linéarité liée aux interactions polariton-exciton permet de contrôler le dispositif en agissant sur une surface de seulement quelques microns carrés avec le faisceau de contrôle, et de réaliser un dispositif dont la taille globale est très réduite (de l'ordre de $20 \mu \mathrm{m}$ ). D'autres dispositifs, tels que des transistors à polaritons, des interrupteurs optiques et des portes optiques ont été réalisés dans différents groupes au niveau international, en utilisant les fortes non-linéarités du système, et sont des briques de base et des démonstrateurs de principe d'une nouvelle plateforme pour le traitement tout optique de l'information.

\section{Superfluidité}

Une autre propriété fascinante des fluides quantiques présentant des interactions est la superfluidité, observée pour la première fois avec des polaritons en 2009 au Laboratoire Kastler Brossel. L'origine de la superfluidité se trouve dans la renormalisation de la courbe de dispersion $\mathrm{E}(\mathrm{k})$, qui se produit lorsque l'énergie d'interaction devient supérieure à l'énergie cinétique des quasi-particules (fig. 3d). La dispersion devient localement linéaire : les excitations du fluide sont alors des ondes " acoustiques » de la densité de particules, et la pente de la courbe de dispersion définit la vitesse de ce "son".
Ce changement de la dispersion entraîne une modification très forte de l'écoulement, en particulier en présence d'un défaut. Considérons ainsi un flux de polaritons de vecteur d'onde $\mathrm{k}$ se propageant dans un système 1D. En présence d'un défaut qui brise localement l'invariance par translation du système (fig. $3 \mathrm{a}$ ), les polaritons peuvent être partiellement rétrodiffusés : en raison de la forme parabolique de la courbe de dispersion, il existe un état quantique de même énergie et de vecteur d'onde opposé à celui des polaritons incidents (fig. 3b). On observe alors des modulations d'intensité en amont du défaut (fig. 3c), qui reflètent les interférences entre les polaritons incidents et les polaritons réfléchis. En revanche, lorsque la dispersion est fortement renormalisée, comme sur la figure $3 \mathrm{~d}$, il n'y a plus aucun état quantique disponible permettant la réflexion élastique des polaritons incidents. Les franges d'interférences disparaissent, ce qui est la signature de la disparition de la rétrodiffusion : c'est le régime superfluide qui est clairement mis en évidence sur la figure 3e. On parle aussi de régime subsonique, car on peut montrer que la superfluidité apparaît lorsque la vitesse du fluide (le flux de polaritons) est inférieure à la vitesse du son. De l'autre côté du défaut, la densité de polaritons est beaucoup plus faible. L'énergie d'interaction devient inférieure à l'énergie cinétique : on est alors dans le régime dit "supersonique».

Imaginons maintenant que des ondes acoustiques de polaritons soient émises en aval du défaut. Comme, dans cette région, le fluide a une vitesse supérieure à celle du son, ces ondes ne peuvent pas remonter le

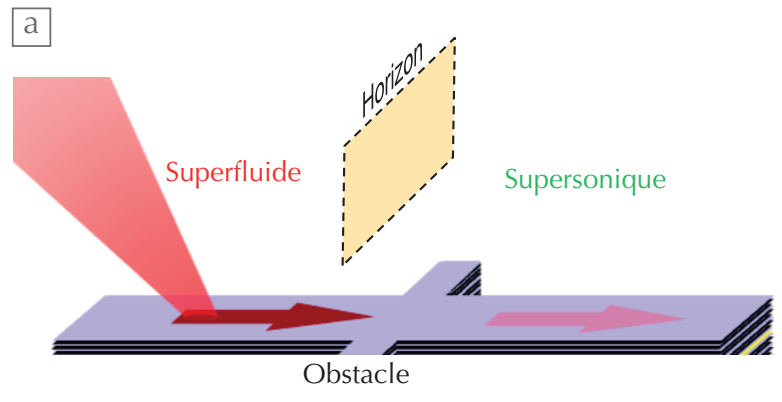

3. Effet d'un obstacle sur la propagation d'un flux de polaritons dans un système 1D.
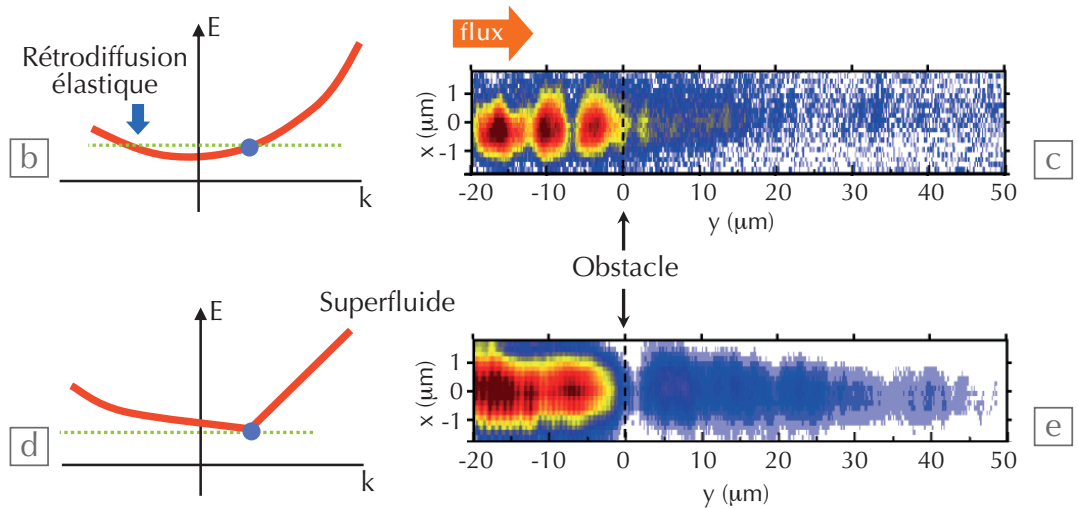

(b) Courbe de dispersion du fluide de polaritons à basse puissance, lorsque les interactions sont négligeables. La dispersion est parabolique et les polaritons sont rétrodiffusés élastiquement quand ils rencontrent l'obstacle, donnant lieu, en amont de l'obstacle, à des oscillations de densité dues à l'interférence entre les polaritons incidents et rétrodiffusés (c). À haute puissance d'excitation optique, les interactions rendent le fluide superfluide et, en amont de l'obstacle, la dispersion devient localement linéaire (d). II n'y a plus d'états disponibles à l'énergie des polaritons incidents et la rétrodiffusion est supprimée : les interférences disparaissent en amont de l’obstacle (e). Dans cette situation, un horizon acoustique est formé. 
$>>$

courant et sont emportées loin du défaut. Elles ne peuvent pas atteindre la région en amont du défaut. Les ondes acoustiques sont donc piégées à la droite du défaut. C'est ce que l'on appelle un « trou noir acoustique ", par analogie aux trous noirs en cosmologie pour lesquels c'est la lumière qui est piégée. L'horizon du trou noir est constitué par le défaut. L'analogie entre l'objet cosmologique et notre réalisation avec des polaritons est en fait très profonde. Et la théorie du trou noir à polaritons prédit même que cet horizon émet de façon spontanée des paquets de polaritons corrélés de part et d'autre de l'horizon, qui sont l'analogue de la radiation de Hawking prédite en 1979 mais encore jamais observée. Un très grand enjeu scientifique aujourd'hui est d'essayer de mesurer ce signal (les prédictions indiquent qu'il est très faible mais peut-être détectable), et de sonder ainsi pour la première fois la radiation de Hawking en laboratoire dans un système analogue.

\section{Gaz de polaritons dans des réseaux, de la suite de Fibonacci au « graphène photonique »}

Les techniques de gravure des microcavités permettent également de réaliser des réseaux aussi bien unidimensionnels que bidimensionnels et de géométries variées. On sculpte alors pour les polaritons un potentiel spatial statique, et les propriétés physiques des polaritons vont refléter les propriétés géométriques et topologiques du réseau. Avec deux exemples, le façonnage d'un potentiel quasi périodique et les polaritons dans un réseau bidimensionnel en nid d'abeilles, nous allons illustrer, dans la suite de l'article, les potentialités de ce système pour émuler des Hamiltoniens complexes, et explorer la physique des fluides quantiques dans des milieux artificiels.

\section{Façonnage d'un potentiel 1D quasi périodique}

Il s'agit d'une séquence qui n'est pas périodique, mais qui présente tout de même (comme les systèmes quasi cristallins) une régularité spatiale particulière, c'est-à-dire des corrélations spatiales à longue distance. Dans le cas de nos microcavités à semiconducteurs, nous avons réalisé un potentiel unidimensionnel selon la suite de Fibonacci. Pour ce faire, nous avons varié la dimension latérale d'une cavité 1D en suivant cette séquence mathématique
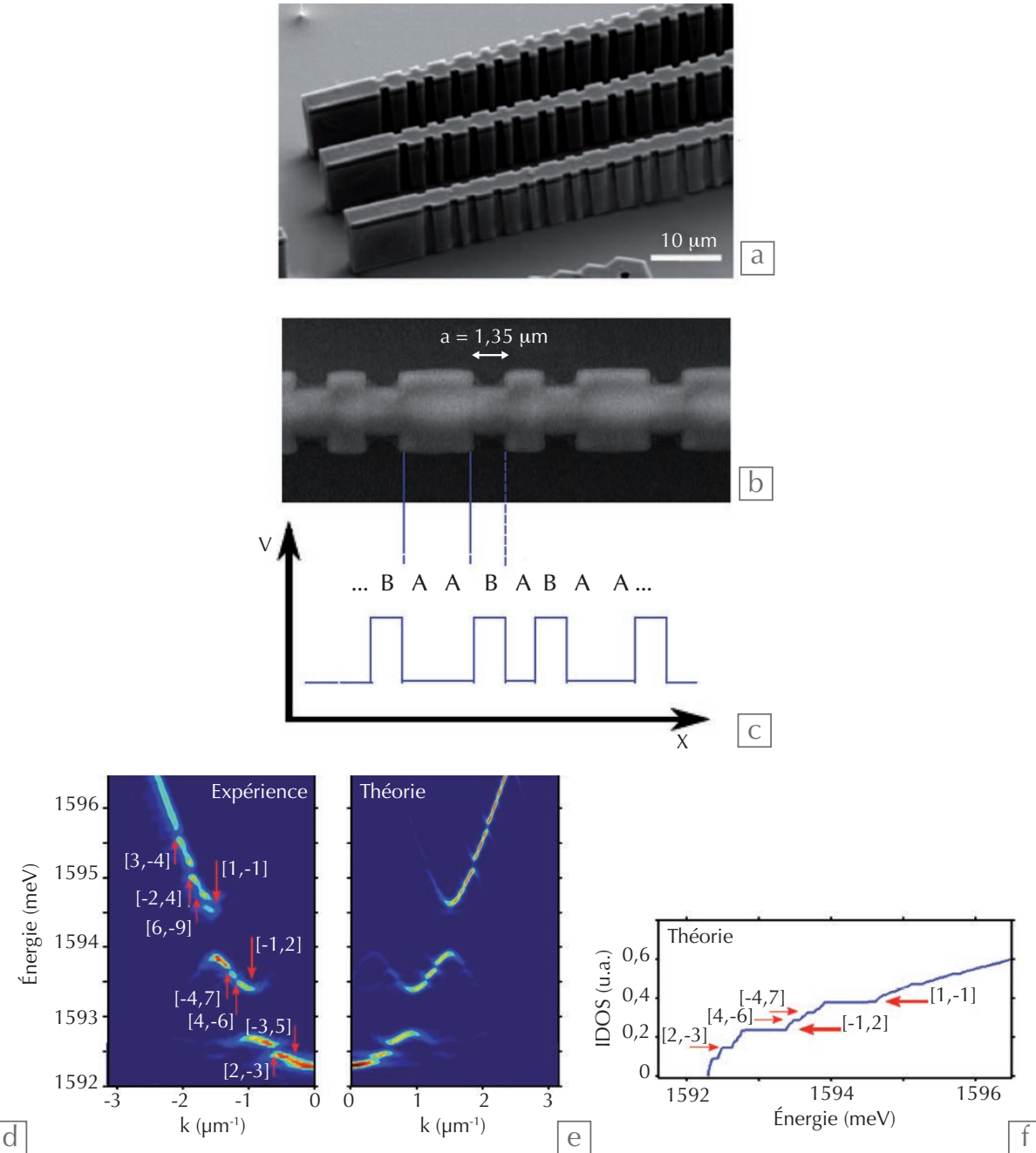

4. Polaritons dans un potentiel 1D quasi périodique. (a) Image en microscopie électronique à balayage de trois cavités 1D présentant une modulation apériodique de leur largeur ; ces cavités sont étudiées individuellement. (b) Image vue du dessus de l'une des cavités 1D. (c) La modulation donne lieu à un potentiel pour les polaritons en forme de « mot » de Fibonacci. Le potentiel de Fibonacci montré sur (a) correspond au mot n=13, et donne lieu à une dispersion fractale, comme montré expérimentalement sur (d) et théoriquement sur (e). La densité d'états intégrée calculée (f) montre une structure auto-similaire, avec des gaps qui se répètent à des échelles différentes.

(fig. 4a-b-c). Ceci génère pour les polaritons un potentiel quasi périodique qui va modifier leurs niveaux d'énergie. On peut construire cette suite à partir des " mots" de Fibonacci. Ils sont formés à partir de deux lettres, A et B, constituées de sections de la cavité $1 \mathrm{D}$ de même longueur et de deux largeurs différentes. Pour construire un mot de Fibonacci avec n lettres, il suffit de coller l'un après l'autre les mots avec n-1 et $n-2$ lettres. Les deux premiers mots ont une seule lettre (A et $B$ ), le mot $n=3$ est $\mathrm{BA}$, le mot $\mathrm{n}=4$ est $\mathrm{BAB}$, etc.

Les bandes d'énergie, mesurées par photoluminescence sur cet échantillon quasi périodique (fig. $4 \mathrm{~d}$ ), sont parfaitement reproduites par la théorie jusque dans leurs moindres détails (fig. 4e). Elles montrent une structure complexe, avec l'ouverture de nombreuses bandes interdites. Chacune d'elles correspond à un vecteur d'onde $\mathrm{k}$ tel qu'il existe deux entiers $p$ et $q$ pour lesquels $\mathrm{k}=(p+q / \sigma) \pi / a$, où $\sigma=(1+\sqrt{5}) / 2$ est le nombre d'or et $a$ la taille des motifs élémentaires de la séquence de Fibonacci. Ainsi, le spectre des états de polaritons reflète directement les propriétés mathématiques de la série de Fibonacci.

Ceci est encore plus visible si l'on s'intéresse à la densité d'états intégrée (IDOS), qui peut être mesurée expérimentalement. Sur la figure 4f, qui montre l'IDOS calculée, chaque plateau correspond à une bande interdite et, entre ces plateaux, se trouvent les différentes mini-bandes d'énergie. Il apparaît trois bandes principales, séparées 
par deux bandes interdites. Mais, si l'on regarde par exemple la bande centrale, on voit qu'elle est elle-même constituée de trois bandes principales séparées par deux bandes interdites; et ainsi de suite. Cette propriété d'autosimilarité reflète le caractère fractal du spectre de polaritons ainsi implémenté. Nous avons pu démontrer que la fractalité apparaît également dans les spectres expérimentaux. Le nombre de classes de taille observé expérimentalement est limité par la largeur spectrale des polaritons : pour en voir davantage, il faut augmenter le temps de vie de ces derniers.

Grâce à cette réalisation expérimentale, il est maintenant possible d'étudier les propriétés physiques de paquets d'ondes de courbes de dispersion non linéaires dans un environnement de dimension spectrale non entière : de nombreuses questions fondamentales relatives à la localisation, au rôle des interactions, au déclin de la cohérence spatiale sont à explorer, aussi bien du point de vue théorique qu'expérimental.

\section{Polaritons dans un réseau bidimensionnel en nid d'abeilles formé par des micropiliers}

Cette géométrie de réseau (fig. 5a) est la même que celle de l'arrangement périodique des atomes de carbone d'un feuillet de graphène. Ce matériau est étudié en ce moment en particulier pour ses propriétés électroniques remarquables : les bandes d'énergie y présentent des cônes correspondant à des relations de dispersion localement linéaires. Au voisinage de chaque cône, l'équation qui décrit le mouvement des électrons a la forme de la célèbre équation relativiste de Dirac, d'où l'appellation " cônes de Dirac ". Ces dispersions particulières sont responsables de propriétés de transport tout à fait remarquables du graphène.

Comme la structure de bandes reflète de façon directe la géométrie et la topologie du réseau, nous pouvons simuler la physique du graphène grâce à notre réseau en nid d'abeilles. Ceci est illustré sur la figure $5 b$ qui montre la structure de bandes mesurée pour les polaritons. Elle révèle directement les « cônes de Dirac " avec leur dispersion linéaire caractéristique. De nombreux effets physiques prédits pour le graphène sont en cours d'étude actuellement avec ce système analogue à polaritons. Des prédictions théoriques très intéressantes concernent aussi les propriétés physiques d'un tel réseau lorsqu'on le déforme. Il va donc être
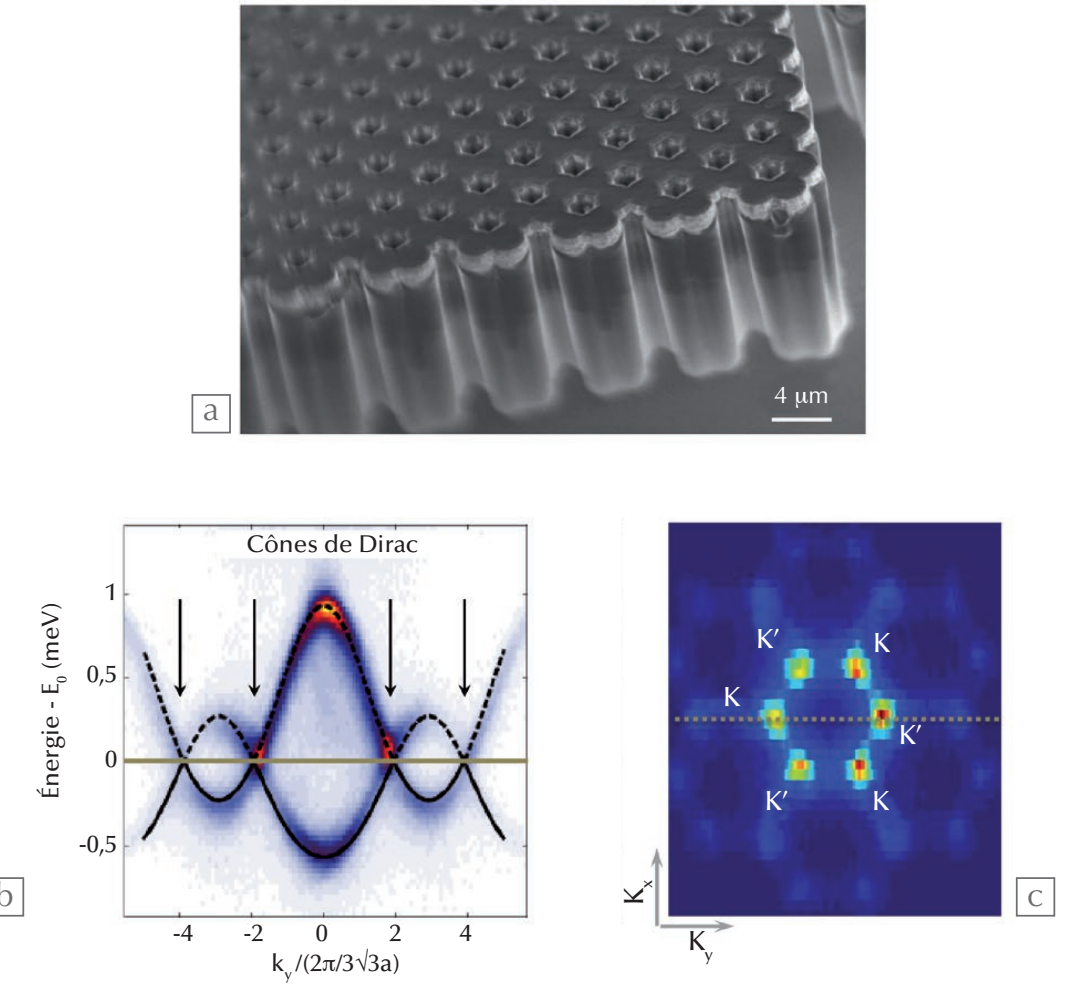

5. Polaritons dans un réseau 2D en nid d'abeilles.

(a) Image en microscopie électronique à balayage d'un réseau de micropiliers en nid d'abeille.

(b) Les courbes de dispersion des polaritons montrent des cônes de Dirac (indiqués par des flèches), équivalents à ceux trouvés pour les électrons dans le graphène.

(c) Émission dans l'espace des impulsions mesurée à l'énergie des points de Dirac : on distingue la première zone de Brillouin du réseau et les zones adjacentes.

possible de tester ces prédictions théoriques en utilisant des polaritons dans des structures photoniques artificielles.

\section{Perspectives}

Le domaine des polaritons de cavité s'est considérablement enrichi depuis leur découverte en 1992. Au départ nouvelles sources de lumière cohérente et objets d'étude en soi, ces fluides de lumière sont aujourd'hui un outil pour concevoir et fabriquer des systèmes analogues. Ils offrent une plateforme façonnable à volonté pour réaliser des réseaux de forme

\section{Bibliographie}

- I. Carusotto et C. Ciuti, "Quantum fluids of light", Rev. Mod. Phys. 85 (2013) 299 (arXiv:1205.6500).

- E. Wartz et al., "Spontaneous formation and optical manipulation of extended polariton condensates", Nature Physics 6 (2010) 860 (arXiv:1004.4084).

- T. Jacqmin et al., "Direct observation of Dirac cones and a flatband in a honeycomb lattice for polaritons", Phys. Rev. Lett. 112 (2014) 116402 (arXiv:1310.8105). complexe et implémenter des Hamiltoniens très divers. Ils permettent d'explorer des effets physiques d'une très grande diversité comme la localisation dans des géométries complexes, des effets topologiques ou encore la superfluidité et les trous noirs ! Les polaritons n'ont certainement pas dit leur dernier mot...

Remerciements

Les auteurs tiennent à remercier tous les collaborateurs qui ont participé à ces travaux : les ingénieurs et techniciens du LPN qui élaborent les échantillons ; les étudiants, doctorants et post-doctorants qui ont réalisé et analysé les expériences, et les collègues théoriciens qui ont aidé au développement de cette physique.
- D. Tanese et al., "Fractal energy spectrum of a polariton gas in a Fibonacci quasi-periodic potential", Phys. Rev. Lett. 112 (2014) 146404 (arXiv:1311.3453).

- H.S. Nguyen et al., "Acoustic Black Hole in a Stationary Hydrodynamic Flow of Microcavity Polaritons", Phys. Rev. Lett. 114 (2015) 036402 (arXiv:1507.04704). 